\title{
Implementaçâo dos Centros de Especialidades Odontológicas no contexto da regionalização da saúde no estado do Ceará, Brasil
}

\author{
I ${ }^{1}$ Cesar Luiz Silva Junior, ${ }^{2}$ Patty Fidelis de Almeida, ${ }^{3}$ Moacir Tavares Martins Filho, \\ ${ }^{4}$ Luciana Dias de Lima I
}

Resumo: O estudo tem como objetivo analisar a implementação regional dos Centros de Especialidades Odontológicas (CEO) no estado do Ceará. Tratase de pesquisa avaliativa baseada em estudo de caso com abordagem qualitativa. Realizaram-se entrevistas semiestruturadas com 13 informantes-chave da gestáo e da academia, complementadas por análise documental e observação não participante, no ano de 2018. A análise de conteúdo considerou o processo de regionalização e a trajetória da política de saúde bucal no estado. Observaram-se forte indução e liderança da Secretaria de Estado da Saúde, que permitiram a inserção da saúde bucal no processo de regionalizaçấo da atenção à saúde. Houve implantaçáo em larga escala de CEO Regionais, em contraposição à diretriz municipalista induzida pela política federal. Ressaltam-se como fatores facilitadores: a expertise dos atores estaduais envolvidos na condução da política, a antecipação da política estadual de saúde bucal às diretrizes nacionais e a implantação dos Consórcios Públicos de Saúde responsáveis pela organização e oferta dos serviços especializados nas regiôes de saúde. A experiência do Ceará sugere a importância do enfoque regional para fortalecimento e consolidação do acesso integral à saúde bucal no âmbito do Sistema Único de Saúde.

Palavras-chave: saúde bucal; políticas públicas de saúde; Planejamento Regional de Saúde; especialidades odontológicas; Brasil.

\author{
1 Programa de Pós-Graduação em \\ Saúde Coletiva, Instituto de Saúde \\ Coletiva, Universidade Federal \\ Fluminense. Niterói-RJ, Brasil \\ (cesarluiz54@gmail.com). ORCID: \\ 0000-0002-1189-6004 \\ 2 Instituto de Saúde Coletiva, \\ Universidade Federal Fluminense. \\ Niterói-RJ, Brasil (pattyfidelis@ \\ id.uff.br). ORCID: 0000-0003- \\ 1676-3574. \\ ${ }^{3}$ Faculdades de Farmácia, \\ Odontologia e Enfermagem, \\ Universidade Federal do \\ Ceará. Fortaleza-CE, Brasil \\ (moacirtavares@yahoo.com.br). \\ ORCID: 0000-0002-3305-8036 \\ ${ }^{4}$ Escola Nacional de Saúde Pública \\ Sergio Arouca, Fundação Oswaldo \\ Cruz. Rio de Janeiro-RJ, Brasil \\ (luciana@ensp.fiocruz.br). ORCID: \\ 0000-0002-0640-8387
}

Recebido em: 11/01/2020 Aprovado em: 09/06/2020 Revisado em: 05/10/2020 


\section{Introdução}

As condições de saúde bucal são internacionalmente reconhecidas como importantes problemas de saúde pública em função da sua prevalência, gravidade, impacto individual e coletivo. Entretanto, uma série de desafios persiste para seu enfrentamento (ANTUNES et al., 2016). Mesmo em países com sistemas públicos e universais, a atenção à saúde bucal não integra, necessariamente, a rede de cuidados em saúde, existindo importantes restriçôes quanto ao acesso e ao modelo de organização. Na Espanha, o Servicio Nacional de Salud, embora apresente ampla carteira, em saúde bucal oferta alguns serviços básicos para adultos como atendimentos de emergência e extraçóes dentárias (BRAVO et al., 2015). No Canadá, a falta de acesso aos serviços odontológicos e o isolamento da atenção geral são desafios (MAIN; LEAKE; BURMAN, 2006). Na França, ainda que acessível para a maioria da população, a saúde bucal é eminentemente curativa, contribuindo para a manutenção das desigualdades (PEGON-MACHAT et al., 2016). Na Inglaterra, após processo recente de reforma, os novos contratos odontológicos incluíram um componente que restringe o acesso aos serviços integrais, o que desafia princípios básicos do National Health Service (LAVERTY, HARRIS, 2018).

No Brasil, a partir dos anos 2000, normas e diretrizes nacionais promoveram a ampliação de açôes e serviços públicos de saúde bucal, em um contexto de históricas desigualdades e predomínio da assistência privada e curativa (NARVAI, 2008). Estudos demonstram avanços no âmbito do Sistema Único de Saúde (SUS) decorrentes dessas políticas, sobretudo pela inserção das equipes de saúde bucal na Estratégia Saúde da Família (ESF) e pela implantação de serviços especializados previstos nas diretrizes da Política Nacional de Saúde Bucal (PNSB) (BRASIL, 2004). Nesse cenário, destacam-se os Centros de Especialidades Odontológicas (CEO) como referência de atenção especializada para as equipes de Atenção Primária à Saúde (APS), tendo em vista a garantia do cuidado integral à saúde bucal (BRASIL, 2018).

Vários estudos sugerem desafios relacionados à descentralização e à regionalização no SUS, sobretudo quanto à capacidade dos municípios em prover o cuidado integral e responder às necessidades em saúde das populaçôes (LIMA et al., 2012; VIANA et al., 2017), desafios estes que se estendem aos CEO. A maior parte dos estudos relacionados aos CEO concentra-se na avaliação de desempenho, limitando-se a 
abordagens sobre o alcance de metas de produtividade e utilização de especialidades (ANDRADE, 2016; PINTO, 2014; CHAVES et al., 2011). Em contrapartida, é essencial reforçar que os desafios da provisão de atenção integral em saúde bucal decorrem de um conjunto complexo de fatores, não exclusivamente relacionados à oferta de procedimentos especializados. Entre eles, destacam-se as dificuldades no acesso à saúde bucal no sistema público de saúde (CONTARATO, 2011) e os problemas de organização e funcionamento da rede de atenção (CHAVES et al., 2010). Ademais, estudos indicam a importância do planejamento da saúde bucal em âmbito regional (VIEIRA, 2014; SILVA et al., 2017; MELLO et al., 2014), ainda que as diretrizes nacionais tenham fortalecido perspectiva da gestão municipal.

O estado do Ceará se destaca historicamente por experiências avançadas na implantação de políticas públicas de saúde com forte tradição na descentralização e regionalização do SUS (LIMA; VIANA, 2011; GOYA et al., 2016), sendo um dos estados pioneiros na implementação do CEO em perspectiva regional. Segundo Lima et al. (2012), o contexto político-institucional expressa a trajetória das políticas de saúde, o modo de funcionamento das instituiçôes e o papel desempenhado pelas organizaçóes envolvidas. Considerando a vertente municipalista que caracterizou as diretrizes da PNSB (CHAVES, 2016) e certa escassez de produçôes científicas sobre a inserção da saúde bucal no contexto da regionalização da saúde, o presente artigo tem por objetivo analisar a implementação regional dos Centros de Especialidades Odontológicas no Ceará, Brasil, no contexto do processo de regionalização e da trajetória da política de saúde bucal no estado.

\section{Métodos}

Trata-se de pesquisa avaliativa, consistindo em estudo de caso com abordagem qualitativa, com utilização de distintas fontes de evidências. Foram realizadas 13 entrevistas semiestruturadas, sendo sete com diretores de CEO Regionais (CEO-R), duas com gestores da Secretaria da Saúde do Estado (SESA-CE) e quatro com acadêmicos de Instituiçóes de Ensino Superior do estado. Todos os entrevistados, tanto da academia quanto da gestão, exerciam ou haviam exercido cargos de gestão/gerência ou assistência na área de saúde bucal ou realizado investigaçôes no campo, identificados em fase exploratória do estudo. As entrevistas, que tiveram duração aproximada de uma hora cada, foram gravadas em aparelho de áudio e 
complementadas por análise documental e observação não participante por meio de visita realizada a um CEO-R e participação no "IV Encontro Estadual de Saúde Bucal do Ceará”, promovido pelo Núcleo de Atenção à Saúde Bucal da SESA-CE. Durante o Encontro, todos os diretores de CEO-R presentes foram convidados a participar da pesquisa, sendo que sete aceitaram realizar entrevista. $\mathrm{O}$ trabalho de campo foi realizado em abril de 2018.

A análise documental abrangeu documentos públicos, disponíveis nos sites da SESA-CE e do Ministério da Saúde como Resoluçôes, Portarias, Leis, Decretos e Manuais do período de 1998, ano da publicação do documento "Diretrizes da regionalização do estado do Ceará” (CEARÁ, 1998), que norteou a institucionalização do sistema de base regional, com influência técnica e política, até o ano de 2018.

Os entrevistados foram identificados por códigos alfanuméricos. Para diretores de CEO-R, utilizou-se a letra "D” e o número sequencial de realização das entrevistas; para gestores estaduais "E", e para Academia a letra "A", seguidas dos respectivos números. O quadro 1 apresenta o perfil dos entrevistados, com destaque para a formação e experiência em diversos âmbito do SUS.

Quadro 1. Perfis dos informantes-chave entrevistados. Fortaleza-CE, 2018

\begin{tabular}{|c|c|c|c|c|}
\hline CÓDIGO & PÓS-GRADUAÇÃO & $\begin{array}{c}\text { FORMAÇÃO } \\
\text { NA ESP-CE }\end{array}$ & GESTÃO SUS & $\begin{array}{c}\text { ASSISTÊNCIA } \\
\text { SUS }\end{array}$ \\
\hline D01 & $\begin{array}{l}\text { Esp.: Endodontia; Gestão } \\
\text { Serviços de Saúde; Gestão da } \\
\text { Clínica }\end{array}$ & Sim & $\begin{array}{l}\text { Diretor Geral } \\
\text { CEO-M Coordenador } \\
\text { Municipal SB }\end{array}$ & PSF \\
\hline D02 & - & Não & $\begin{array}{l}\text { SMS; } \\
\text { Sub-SMS. }\end{array}$ & $\begin{array}{l}\text { Central } \\
\text { Abastecimento } \\
\text { Farmacêutico }\end{array}$ \\
\hline D03 & $\begin{array}{l}\text { Esp.: Ortodontia; Gestão } \\
\text { Serviços de Saúde } \\
\text { Mestrado Ortodontia }\end{array}$ & Sim & $\begin{array}{l}\text { Diretora } \\
\text { Administrativo- } \\
\text { Financeiro CEO-R }\end{array}$ & $\begin{array}{l}\text { CEO } \\
\text { PSF }\end{array}$ \\
\hline D04 & $\begin{array}{l}\text { Esp.: Gestão Serviços } \\
\text { de Saúde; Ortodontia; } \\
\text { Endodontia; Periodontia }\end{array}$ & Sim & $\begin{array}{l}\text { Coordenador } \\
\text { Municipal SB } \\
\text { SMS } \\
\text { Coordenador CEO-M }\end{array}$ & PSF \\
\hline D05 & $\begin{array}{l}\text { Esp.: Gestão Serviços de } \\
\text { Saúde; Ortodontia } \\
\text { Mestrado Gestấo em Saúde }\end{array}$ & Sim & $\begin{array}{l}\text { Diretora Geral } \\
\text { CEO-R; Coordenadora } \\
\text { Municipal SB }\end{array}$ & PSF \\
\hline
\end{tabular}

continua... 


\begin{tabular}{|c|c|c|c|c|}
\hline CÓDIGO & PÓS-GRADUAÇÃO & $\begin{array}{c}\text { FORMAÇÃO } \\
\text { NA ESP-CE }\end{array}$ & GESTÃO SUS & $\begin{array}{c}\text { ASSISTÊNCIA } \\
\text { SUS }\end{array}$ \\
\hline D06 & $\begin{array}{l}\text { Aperf.: Cirurgia e Implante } \\
\text { Esp.: SF; Gestấo Serviços de } \\
\text { Saúde }\end{array}$ & Sim & $\begin{array}{l}\text { Coordenador } \\
\text { Municipal SB } \\
\text { Gestão de Projetos }\end{array}$ & PSF \\
\hline D07 & $\begin{array}{l}\text { Esp.: Endodontia; Cirurgia } \\
\text { Oral Menor }\end{array}$ & Não & $\begin{array}{l}\text { Coordenador } \\
\text { Municipal SB }\end{array}$ & PSF \\
\hline E01 & $\begin{array}{l}\text { Mestrado e Doutorado Saúde } \\
\text { Pública }\end{array}$ & Não & $\begin{array}{l}\text { CRO-CE; } \\
\text { Gabinete Prefeitura; } \\
\text { SMS; } \\
\text { Gabinete Governador; } \\
\text { Diretor de Pós } \\
\text { Graduaçáo }\end{array}$ & $\begin{array}{l}\text { Cirurgião- } \\
\text { dentista SUS }\end{array}$ \\
\hline E02 & $\begin{array}{l}\text { Esp.: Periodontia: Gestão } \\
\text { formada em Evidências; } \\
\text { Redes em Atenção em Saúde; } \\
\text { Regulação em Saúde } \\
\text { Residência em SF } \\
\text { Mestrado Educação em } \\
\text { Ciências da Saúde }\end{array}$ & Sim & $\begin{array}{l}\text { Coordenador } \\
\text { Municipal SB } \\
\text { Secretário Municipal } \\
\text { Adjunto de Saúde } \\
\text { Coordenador estadual } \\
\text { SB }\end{array}$ & $\begin{array}{l}\text { CEO-R } \\
\text { CEO-M }\end{array}$ \\
\hline A01 & $\begin{array}{l}\text { Esp.: Gestáo em Saúde } \\
\text { Residência Odontopediatria } \\
\text { Mestrado Odontopediatria e } \\
\text { em Saúde Pública } \\
\text { Doutorado Saúde Pública }\end{array}$ & Sim & $\begin{array}{l}\text { Cargos na Gestão } \\
\text { Estadual; } \\
\text { Gerência CEO-M; } \\
\text { Coordenadora Estadual } \\
\text { SB }\end{array}$ & UBS \\
\hline A02 & $\begin{array}{l}\text { Esp.: Saúde Pública } \\
\text { Mestrado e Doutorado } \\
\text { Odontologia Preventiva e } \\
\text { Social }\end{array}$ & Não & $\begin{array}{l}\text { Coordenadora Estadual } \\
\text { do PMAQ }\end{array}$ & UBS \\
\hline A03 & $\begin{array}{l}\text { Mestrado Odontologia } \\
\text { Preventiva e Social; } \\
\text { Doutorado Ciências da } \\
\text { Saúde/Saúde Pública }\end{array}$ & Não & Não & PSF \\
\hline A04 & $\begin{array}{l}\text { Aperf.: Gestão em Saúde; } \\
\text { Mestrado e Doutorado em } \\
\text { Saúde Coletiva } \\
\text { Esp.: Odontologia SF; Gestáo } \\
\text { Serviços de Saúde; Auditoria } \\
\text { em Serviços de Saúde }\end{array}$ & Sim & $\begin{array}{l}\text { Coordenador } \\
\text { Municipal SB } \\
\text { Diretor Geral CEO-R. }\end{array}$ & PSF \\
\hline
\end{tabular}

Fonte: elaboração própria, a partir das entrevistas, 2018.

Siglas: CEO-M - Centro de Especialidades Odontológicas Municipal; CEO-R - Centro de Especialidades Odontológicas Regional; ESP-CE - Escola de Saúde Pública do Ceará; PSF - Programa Saúde da Família; SB - Saúde Bucal; SMS - Secretário Municipal de Saúde; CRO - Conselho Regional de Odontologia. 
A análise do material empírico foi realizada considerando dois eixos: (1) o processo de regionalização da atenção à saúde no estado; e (2) a trajetória da política de saúde bucal, sendo utilizada matriz produzida a partir dos estudos de Contarato (2011), Lima e Viana (2011) e Vieira (2014) apresentadas no quadro 2 na seção resultados, que também sumariza alguns achados do estudo.

Os dados foram organizados e analisados com base nas categorias da matriz de análise. Orientadas pela análise de conteúdo temática (BARDIN, 2011) e suas respectivas etapas, informaçóes convergentes foram trianguladas, buscando articular a perspectiva resultante das diferentes técnicas de pesquisa e dos diversos sujeitos e fontes do estudo. Todos os dados passaram por análise de conteúdo vertical considerando-se em um primeiro momento as dimensôes de análise e o posicionamento de cada informante-chave e, posteriormente, por leitura horizontal comparando-se e confrontando-se as perspectivas dos atores e demais fontes de informação.

A pesquisa foi aprovada pelo Comitê de Ética em Pesquisa da Universidade Federal Fluminense (CEP/UFF) sob parecer n. 2.207.909.

\section{Resultados}

Foi no governo Tasso Jereissati (1995-1998 / 1999-2002), denominado "eficientista / tecnicista" (A01), que a regionalização na saúde no Ceará ganhou força, a partir de processos de aprendizagem institucional. Consultorias nacionais e internacionais, especialmente da Organização Pan-americana da Saúde (OPAS) e do Banco Mundial, influenciaram os atores sociais da SESA-CE e dos municípios em relação a estratégias de microrregionalização, cujo principal objetivo era a melhoria do acesso aos serviços de saúde (E02). A Escola de Saúde Pública do Ceará, instituição vinculada à SESA-CE, destacou-se pelo importante papel de apoio pedagógico e operacional para capacitação dos profissionais e gestores da saúde, sendo o principal agente formador para o SUS no estado (A02).

O documento "Diretrizes de Reorganização da Atenção e dos Serviços do Sistema Único de Saúde do Ceará” (CEARÁ, 1998), influenciado pelas consultorias internacionais, reafirmou a diretriz da regionalização como norteador de um sistema de saúde de base regional, mas não mencionava ou incluía as açôes de saúde bucal, o que de certa forma intensificou as disputas internas na SESA-CE. Naquele momento, era fundamental reconhecer e explicitar a saúde bucal no processo de organização 
do sistema de saúde, considerando que a não inclusão representaria perdas históricas (A01; A03). A partir de articulaçóes e disputas no Conselho Estadual de Saúde, a Resolução no 06/1998 (CEARÁ, 19981) representou um marco ao complementar o documento das "Diretrizes" (CEARÁ, 1998) e possibilitar a entrada da saúde bucal na agenda de prioridades do estado.

Para além da elaboração do primeiro Plano Diretor de Regionalização (PDR) em 1998, que teve o documento marco (CEARÁ, 1998) como base orientadora, e suas revisôes nos anos de 2001, 2006, 2010 e 2014, o processo de regionalização no estado não se limitou à reorganização dos territórios, mas também contribuiu para a diminuição da dependência dos municípios do interior em relação à capital, com o fortalecimento das instâncias de representação, pactuação e negociação intergovernamental (E01), como a Comissão Intergestores Bipartite e Comissões Intergestores Regionais (CIR). Destacaram-se também as estruturas descentralizadas de gestão da SESA-CE, as chamadas Coordenadorias Regionais de Saúde (CRES), caracterizadas como os "olhos do Estado" (D06), responsáveis por estimular as discussôes regionais em parceria com os gestores municipais.

No governo Lúcio Alcântara (2003-2006), informantes da academia reforçaram a relevância do papel da SESA-CE para a expansão e qualificação da APS, por meio da ampliação das equipes de saúde bucal no "Programa Dentista da Família", e da atenção especializada, através da implantação dos CEO-R no estado. A figura 1 resume as etapas da política estadual de saúde bucal, destacando marcos nacionais e estaduais no período anterior às diretrizes da Política Nacional de Saúde Bucal (1990-2003). 
Figura 1. Linha do Tempo - marcos nacionais e estaduais da política de saúde bucal 1990 a 2003 e 2004 a 2018. Fortaleza-CE, 2018
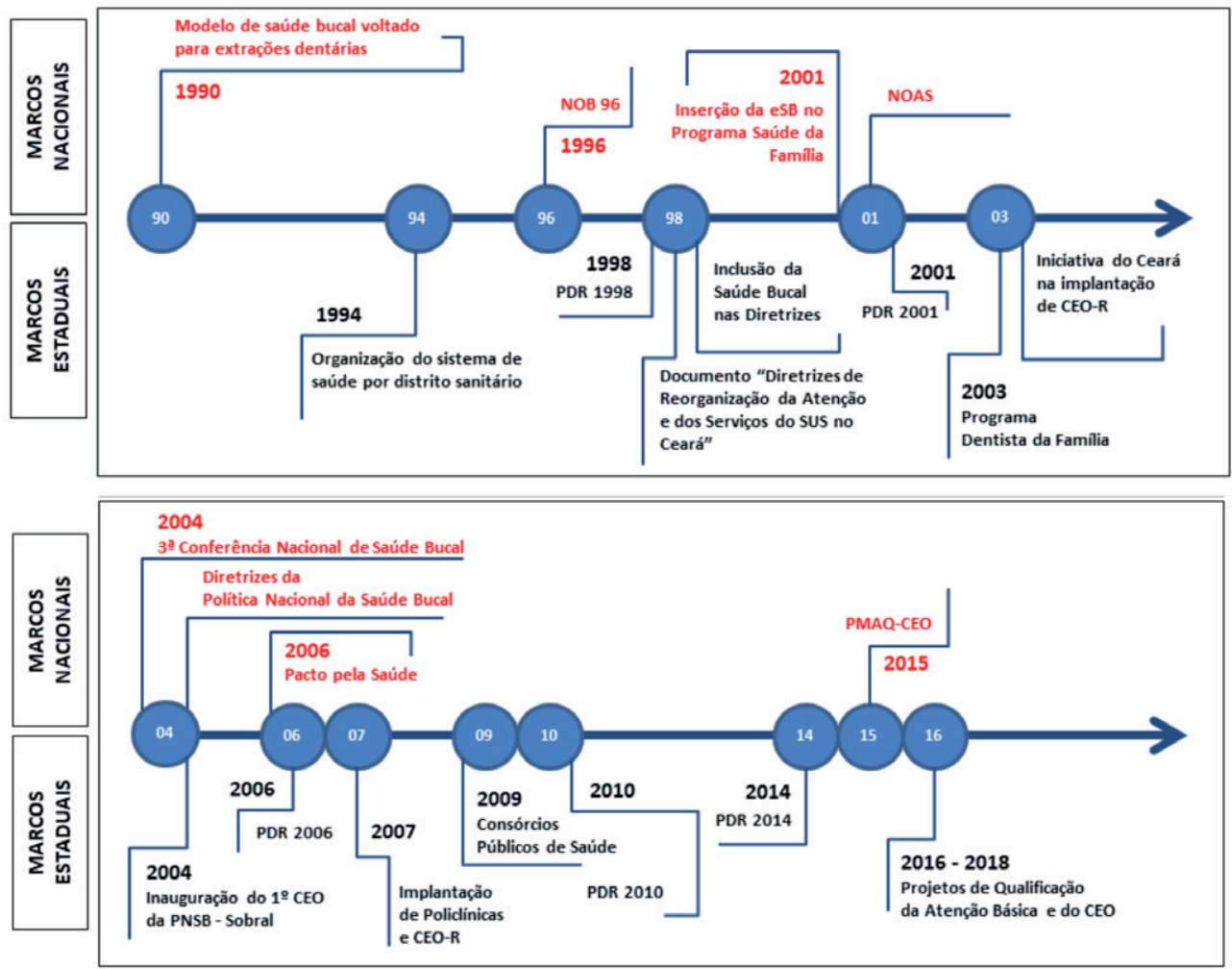

Fonte: elaboração própria, 2018.

Em 2004, foi lançada a Política Nacional de Saúde Bucal (PNSB) (BRASIL, 2004) que apresentou conjunto de açôes para reorganizar os serviços de saúde bucal em todos os níveis de atenção nos diversos territórios, incluindo os Centros de Especialidades Odontológicas, como estratégia relevante para alcance da integralidade do cuidado. Nesse contexto, o estado já apresentava CEO na capital Fortaleza desde 1965, sendo: CEO Centro (desde 1965), CEO Joaquim Távora (desde 1994) e CEO Rodolfo Teófilo (desde 1999) (A01; A03). A forte vertente municipalista da PNSB (BRASIL, 2004), segundo avaliação de informante-chave, se expressou em aumento significativo do número de CEO municipais em todo país, inclusive no Ceará, desconsiderando por muitas vezes, a organização da APS, as necessidades de saúde e a sustentabilidade dos aportes financeiros municipais. 
Avaliou-se que as diretrizes da PNSB (BRASIL, 2004) não reconheceram o potencial das experiências até então desenvolvidas no estado, que priorizava a lógica regional. Alguns informantes da academia apontaram o antagonismo político entre governo estadual (Partido da Social-Democracia Brasileira - PSDB) e governo federal (Partido dos Trabalhadores - PT) à época justificou tal posicionamento. Ainda assim, a expertise, conforme pode ser observado no quadro 1, e a articulação dos atores estaduais junto ao governo federal foram importantes, tendo o Ceará como cenário da inauguração do $1^{\circ} \mathrm{CEO}$ do Programa Brasil Sorridente, em Sobral (A01; E02) no ano de 2004. Até o ano de 2006, foram implantados mais cinco CEO-R no estado (Aracati, Tauá, São Gonçalo, Crato e Iguatú) (A03).

Na gestão Cid Gomes (2007-2010/2011-2014), a trajetória da política de saúde bucal sofreu modificações. O governo estadual intensificou a adoção de estratégias que contribuíram significativamente para a ampliação do acesso e da qualidade dos serviços especializados na lógica regional, com o Programa de Expansão e Melhoria da Assistência Especializada à Saúde (PROEXMAES) e o Monitoramento e Acompanhamento dos Projetos Prioritários (MAPP) da Saúde (E02), ferramenta informatizada que permitia à gestão organizar e acompanhar a execução financeira de projetos.

A partir de 2009, sob liderança do estado, iniciou-se a implantação dos Consórcios Públicos de Saúde (CPS), responsáveis pela administração dos CEO-R e das policlínicas de especialidades nas regiōes de saúde (E01; A04). Em 2012, o Ceará contava com 21 CPS nas 22 regiôes, exceto na regional de Fortaleza, consolidando sua posição inovadora pela implantação em larga escala. Foi destacado que a participação ativa os diretores de CEO-R nas reuniôes dos consórcios e da CIR (e na antiga Comissão Gestora Regional - CGR) favoreceu avanços na representatividade da saúde bucal nas instâncias gestoras do SUS.

Entre os documentos para a operaçáo dos CPS, o Contrato de Rateio define a contrapartida de municípios e estado no financiamento das policlínicas e CEO-R. Os $60 \%$ que compunham os aportes municipais eram provenientes do Imposto sobre Circulação de Mercadorias e Serviços (ICMS), descontados na fonte, evitando-se descontinuidades por atrasos ou falta de pagamento (D07). O teto foi definido como $7,5 \%$ do valor total do ICMS municipal, estratégia para não comprometer o orçamento dos municípios com menor arrecadaçáo. Nesses casos, o Estado se responsabilizava pela complementação da contrapartida municipal. Apesar de o governo federal 
não participar do financiamento dos CPS, no caso do $\mathrm{CEO}$, os aportes da PNSB (BRASIL, 2004) compunham o percentual de investimento municipal (A04).

Os Contratos de Programa, a partir de critérios populacionais, definiam as vagas e os serviços ofertados para cada município. Neste aspecto, os diretores de CEO-R reforçaram que o município-polo concentrava a maior parte da oferta destes serviços, além de contar também com CEO municipais. Ainda assim, os informanteschave mencionaram que a lógica cooperativa se sobrepunha às possíveis tensôes. Com melhor infraestrutura e prestação de serviços, os CEO-R logo se tornaram prioridade para os gestores, representando grande mudança de paradigma na saúde pública do estado, embora convivessem, nem sempre de forma articulada, com os CEO municipais, induzidos pelas políticas federais.

Em muitos cenários, a APS permanecia com limitaçōes estruturais e financeiras (A02). Na avaliação de alguns informantes-chave, os CEO-R apresentavam-se como "ilhas" de grande aporte tecnológico em meio a uma APS fragilizada. Também apontaram certo desconhecimento, por parte de gestores municipais, da perspectiva do CEO como componente da rede. Nesse sentido, alguns prefeitos defendiam a ideia de que o CEO-R, isoladamente, seria capaz de atender às necessidades de saúde da regiâo, compensando as deficiências da APS dos seus municípios (A04; D04; D07).

No governo Camilo Santana (2015-2018), os instrumentos de planejamento, norteados pelo Plano Estadual de Saúde (PES) 2016-2019 (CEARÁ, 2016), elencavam a saúde bucal entre os compromissos prioritários na organização das Redes de Atenção à Saúde do estado, com destaque para iniciativas de qualificação dos processos de trabalho na APS e das equipes dos CEO-R, com impacto na melhoria dos indicadores de saúde bucal. A figura 1 apresenta marcos nacionais e estaduais a partir do lançamento das diretrizes da Política Nacional de Saúde Bucal (2004-2018).

Entre os desafios dos CEO-R, a maioria dos entrevistados destacou o processo de "prefeiturizaçáo dos consórcios", cuja presidência é de um dos prefeitos dos municípios consorciados. Foram mencionadas nomeaçóes de cargos para policlínicas e CEO-R sem qualificação técnica; ampliação ou redução de especialidades sem parâmetros epidemiológicos ou sustentabilidade financeira; desigualdades salariais entre profissionais de diferentes Consórcios; deficiências nos processos de monitoramento e avaliação; perda de parâmetros em licitaçôes e mudanças no processo seletivo para diretor decorrentes da ingerência local nos CPS, sobretudo nos períodos de maior distanciamento da SESA-CE (D01; D02; D03; D05; E01). 
Nesta perspectiva, um informante reconheceu que o estado se encontrava em um momento de reaproximação da gestáo dos CPS para planejamento e rediscussão dos processos de trabalho e gestão (E01).

Outro desafio mencionado se referiu à sustentabilidade financeira dos Consórcios, com maiores dificuldades nos últimos anos. Apesar da previsão de reajustes anuais para custeio, desde 2012, teriam sido congelados, mesmo diante da contratualização de novos serviços e aumento dos gastos com pessoal. Com o objetivo de reequilibrar as finanças dos CPS, foram mencionadas estratégias de planejamento e revisão financeira em algumas regiôes, inclusive com devolução do transporte sanitário para administração dos municípios, diminuição de cargos comissionados, entre outras medidas (D07). Ainda assim, avaliou-se que o subfinanciamento ainda não havia impactado a qualidade dos CEO-R.

$\mathrm{Na}$ avaliação de políticas, programas e serviços destaca-se a importância dos fatores contextuais ou influências externas na determinação das intervençóes sociais (CHAMPAGNE et al., 2011). No caso estudado, os principais fatores facilitadores e dificultadores na implementação dos Centros de Especialidades Odontológicas no contexto da regionalização da atenção à saúde no estado do Ceará foram sumarizados no quadro 2, considerando os eixos e as categorias de análise.

Quadro 2. Síntese dos principais fatores facilitadores e dificultadores na implementação dos Centros de Especialidades Odontológicas no contexto da regionalização da atenção à saúde no estado do Ceará. Fortaleza-CE, 2018

\begin{tabular}{|l|l|}
\hline \multicolumn{1}{|c|}{ CATEGORIAS } & \multicolumn{1}{c|}{ FATORES FACILITADORES E DIFICULTADORES } \\
\hline $\begin{array}{l}\text { Desenhos e estratégias } \\
\text { no processo de } \\
\text { regionalização }\end{array}$ & $\begin{array}{l}\text { Facilitador: Processos institucionais a partir de consultorias nacionais e } \\
\text { internacionais / Fortalecimento de arranjos de governança regional. } \\
\text { Dificultador: Tensóes entre o município-polo e os demais da região. }\end{array}$ \\
\hline $\begin{array}{l}\text { Implantação dos } \\
\text { Consórcios Públicos de } \\
\text { Saúde }\end{array}$ & $\begin{array}{l}\text { Facilitador: Formato peculiar quanto à gestáo interfederativa, retenção e } \\
\text { repasse dos recursos; } \\
\text { Dificultador: Centralidade dos prefeitos na configuração dos consórcios } \\
\text { / Subfinanciamento do SUS. }\end{array}$ \\
\hline $\begin{array}{l}\text { Açôes e papeis dos } \\
\text { atores no processo de } \\
\text { regionalização }\end{array}$ & $\begin{array}{l}\text { Facilitador: Forte indução e condução da política pela SESA-CE / } \\
\text { Continuidade administrativa na gestão estadual; } \\
\text { Dificultador: Influência da política local / Distanciamento da SESA-CE. }\end{array}$ \\
\hline
\end{tabular}

continua... 


\begin{tabular}{|c|c|}
\hline $\begin{array}{l}\text { Instrumentos } \\
\text { de Pactuação e } \\
\text { Norteadores no processo } \\
\text { de regionalização }\end{array}$ & $\begin{array}{l}\text { Facilitador: Pioneirismo na elaboração de documentos norteadores, } \\
\text { como as "Diretrizes" e o 1º PDR (1998); } \\
\text { Dificultador: Influência de normas ministeriais à municipalização } \\
\text { atomizada na saúde. }\end{array}$ \\
\hline $\begin{array}{l}\text { Inserção do CEO-R } \\
\text { no processo de } \\
\text { regionalização }\end{array}$ & $\begin{array}{l}\text { Facilitador: Adoção de estratégias para ampliaçáo e expansão da atenção } \\
\text { especializada nas regiốes de saúde; } \\
\text { Dificultador: Ausência da saúde bucal no documento das "Diretrizes". }\end{array}$ \\
\hline $\begin{array}{l}\text { Desenhos e estratégias } \\
\text { da Política de Saúde } \\
\text { Bucal }\end{array}$ & $\begin{array}{l}\text { Facilitador: Antecipação à Política de Saúde Bucal, a partir de estratégias } \\
\text { de expansão da APS e da atenção especializada em perspectiva regional; } \\
\text { Dificultador: Desenho descentralizador (municipalista) das diretrizes da } \\
\text { PNSB. }\end{array}$ \\
\hline $\begin{array}{l}\text { Ações e papeis } \\
\text { dos atores na } \\
\text { implementação da } \\
\text { Política de Saúde Bucal }\end{array}$ & $\begin{array}{l}\text { Facilitador: A notória expertise, formação e articulação dos atores no } \\
\text { estado; ESP-CE como principal aparelho formador; } \\
\text { Dificultador: Discordâncias político-partidárias entre os governos } \\
\text { estadual e federal no lançamento das diretrizes da PNSB. }\end{array}$ \\
\hline $\begin{array}{l}\text { Prioridade da Política } \\
\text { de Saúde Bucal }\end{array}$ & $\begin{array}{l}\text { Facilitador: Protagonismo da SESA-CE no planejamento e na condução } \\
\text { da Política em perspectiva regional; } \\
\text { Dificultador: Priorização da atenção especializada em detrimento da } \\
\text { APS. }\end{array}$ \\
\hline $\begin{array}{l}\text { Inserção do CEO-R } \\
\text { na trajetória da saúde } \\
\text { bucal }\end{array}$ & $\begin{array}{l}\text { Facilitador: Iniciativa pioneira do estado de investir nos CEO-R com } \\
\text { garantia de melhores condiçóes de infraestrutura e prestação de serviços } \\
\text { especializados em saúde bucal; } \\
\text { Dificultador: Desconhecimento das atribuiçóes do CEO na rede de } \\
\text { saúde bucal por parte de alguns gestores municipais. Menor investimento } \\
\text { nas equipes de saúde bucal na APS. }\end{array}$ \\
\hline $\begin{array}{l}\text { Instrumentos } \\
\text { de Pactuação e } \\
\text { Norteadores na } \\
\text { trajetória da saúde } \\
\text { bucal }\end{array}$ & $\begin{array}{l}\text { Facilitador: Inserção da saúde bucal como complemento das "Diretrizes" } \\
\text { / Elaboração de instrumentos norteadores na configuração dos CPS; } \\
\text { Dificultador: Influência das diretrizes da PNSB (2004), com forte } \\
\text { direção municipalista. }\end{array}$ \\
\hline $\begin{array}{l}\text { Estratégias e } \\
\text { mecanismos de } \\
\text { implementação do } \\
\text { CEO-R }\end{array}$ & $\begin{array}{l}\text { Facilitador: Escolha pelos Consórcios Públicos de Saúde como } \\
\text { modalidade de administração; } \\
\text { Dificultador: Implantação de CEO municipais, fortemente induzidos } \\
\text { pela PNSB. }\end{array}$ \\
\hline
\end{tabular}

Fonte: elaboração própria, 2018.

Siglas: APS - Atenção Primária à Saúde; CEO - Centro de Especialidades Odontológicas; CEO-R Centro de Especialidades Odontológicas Regional; CPS - Consórcio Público de Saúde; ESP-CE - Escola de Saúde Pública do Ceará; PDR - Plano Diretor de Regionalização; PNSB - Política Nacional de Saúde Bucal; SESA-CE - Secretaria da Saúde do Estado do Ceará; SUS - Sistema Único de Saúde. 
A partir das subdimensôes e categorias de análise, o quadro 3 apresenta as principais avaliações dos atores na implementação dos Centros de Especialidades Odontológicas no contexto da regionalização da atenção à saúde no estado do Ceará. Como avaliação geral, os informantes reforçaram que as conquistas em saúde bucal no estado, principalmente relacionadas aos CEO-R, alcançaram importante grau de institucionalidade política, que, de certa forma, garantiu a sustentabilidade do serviço mesmo diante de um cenário nacional marcado pelo subfinanciamento das ações e serviços do SUS.

Quadro 3. Avaliação dos atores sobre a implementação dos Centros de Especialidades Odontológicas no contexto da regionalização da atenção à saúde no estado do Ceará. Fortaleza-CE, 2018

\begin{tabular}{|c|c|}
\hline CATEGORIAS & AVALIAÇĀO DOS ATORES \\
\hline $\begin{array}{l}\text { Desenhos e estratégias no } \\
\text { processo de regionalização }\end{array}$ & $\begin{array}{l}\text { "Então, sempre o Ceará foi pioneiro nesse processo de regionalização aqui } \\
\text { no Brasil, porque normalmente tomava a iniciativa de implantar os serviços } \\
\text { atuando com base nas necessidades da população e tentando fazer esta } \\
\text { articulação entre os gestores." (A04). } \\
\text { "Então, com certeza existe uma cooperação. Para mim, um dos principais } \\
\text { papéis, além de levar a média complexidade, foi também de fortalecer a } \\
\text { interação e integraçáo entre os municípios. Junto das CRES, os consórcios } \\
\text { vêm a fortalecer ainda mais a regiáo." (D06). }\end{array}$ \\
\hline $\begin{array}{l}\text { Implantação dos } \\
\text { Consórcios Públicos de } \\
\text { Saúde }\end{array}$ & $\begin{array}{l}\text { "Foi uma grande jogada do governador em atrelar estas unidades regionais } \\
\text { na atenção secundária aos consórcios. Porque, a rigor, os serviços de } \\
\text { maior densidade tecnológica ficam sob a responsabilidade do Estado e os } \\
\text { municípios teriam responsabilidade da atenção básica. Então, os municípios } \\
\text { podem se organizar regionalmente para estar ofertando para aquele } \\
\text { município que tem menor condição." (A04). } \\
\text { "A dificuldade é a gente fazer com que os prefeitos não façam do consórcio } \\
\text { a prefeitura deles, o que a gente tem chamado de 'prefeiturização' dos } \\
\text { consórcios, botando parentes, cabides de emprego, pessoas do município } \\
\text { dele, e que tem atrapalhado às vezes alguns consórcios. Essa 'prefeiturizaçâo' } \\
\text { do consórcio é um grande desafio para os nossos CEOs Regionais." (D05). }\end{array}$ \\
\hline $\begin{array}{l}\text { Ações e papéis dos } \\
\text { atores no processo de } \\
\text { regionalização }\end{array}$ & $\begin{array}{l}\text { "A SESA que deu início, de uma forma pioneira, pelo que eu entendo. [...] } \\
\text { Inclusive com a criaçáo das Coordenadorias Regionais de Saúde em cada } \\
\text { região - tem uma sede que é de responsabilidade do Estado [...] e são eles que } \\
\text { puxam essas discussôes regionais" (D06). }\end{array}$ \\
\hline $\begin{array}{l}\text { Instrumentos de } \\
\text { pactuaçáo e norteadores } \\
\text { no processo de } \\
\text { regionalização }\end{array}$ & $\begin{array}{l}\text { "E, quando a gente teve esse processo a partir das portarias ministeriais e } \\
\text { teve o direcionamento e o incentivo do Ministério para que os municípios se } \\
\text { organizassem nas Redes, acredito que para o estado foi bem mais fácil por ele já } \\
\text { ter essa organizaçāo." (A03). }\end{array}$ \\
\hline
\end{tabular}

continua... 


\begin{tabular}{|c|c|}
\hline $\begin{array}{l}\text { Inserção do CEO-R no } \\
\text { processo de regionalização }\end{array}$ & $\begin{array}{l}\text { "Por outro lado, (o processo de regionalização do Estado) criou uma } \\
\text { condiçấo de oportunidades de a saúde bucal estar na agenda, de se colocar } \\
\text { os CEOs nas regióes, dos dentistas começarem a se mobilizar para isso } \\
\text { acontecer." (A01). }\end{array}$ \\
\hline $\begin{array}{l}\text { Desenhos e estratégias da } \\
\text { Política de Saúde Bucal }\end{array}$ & $\begin{array}{l}\text { "Eu considero o fato também do processo de regionalização ter ajudado a } \\
\text { política de saúde bucal, que favoreceu o desenho da política no estado, nas } \\
\text { regiốes, de forma a incluir a saúde bucal nas redes e linhas de atenção que o } \\
\text { Estado priorizava." (A03). }\end{array}$ \\
\hline $\begin{array}{l}\text { Ações e papéis dos atores } \\
\text { na implementação da } \\
\text { Política de Saúde Bucal }\end{array}$ & $\begin{array}{l}\text { "Foi o documento "Diretrizes da regionalização do estado do Ceará", de } \\
\text { 1998. Ali éramos nós, os poucos que estavam defendendo a saúde bucal e } \\
\text { brigando com os consultores que não queriam colocar e questionando o } \\
\text { processo. [...] A estratégia foi insistir, dizer que a 'saúde bucal precisa estar } \\
\text { aqui'" (A01). }\end{array}$ \\
\hline $\begin{array}{l}\text { Prioridade da Política de } \\
\text { Saúde Bucal }\end{array}$ & $\begin{array}{l}\text { "Quando os CEOs começaram a funcionar, a gente começou a sofrer porque } \\
\text { não tem como o CEO funcionar bem se a atenção básica náo funciona bem. } \\
\text { Entáo, os municípios passaram a querer que o CEO fosse o hospital das } \\
\text { carências" (D07). }\end{array}$ \\
\hline $\begin{array}{l}\text { Inserçáo do CEO-R na } \\
\text { trajetória da saúde bucal }\end{array}$ & $\begin{array}{l}\text { "Víamos que o CEO estadual funcionava de uma maneira melhor e mais } \\
\text { organizada, sem tantas dificuldades em relaçáo aos CEOs municipais. [...] } \\
\text { Além disso, os CEOs municipais às vezes não tinham todas as especialidades } \\
\text { - tinham as cinco especialidades mínimas preconizadas pela Política } \\
\text { Nacional de Saúde Bucal, mas os CEOs estaduais vieram com outras } \\
\text { especialidades." (A02). }\end{array}$ \\
\hline $\begin{array}{l}\text { Instrumentos de } \\
\text { Pactuaçáo e Norteadores } \\
\text { na trajetória da saúde } \\
\text { bucal }\end{array}$ & $\begin{array}{l}\text { "Eu digo que foi muito mais pelo viés político do Ministério seguir na sua } \\
\text { política de articular diretamente com município [...], se manteve com a } \\
\text { política de saúde bucal. [...] É só questão de financiamento." (A01). } \\
\text { "Então, por a gente ter contrato, eu tenho as obrigaçōes. Como por exemplo, } \\
\text { eu tenho a obrigaçáo de ofertar } 262 \text { vagas de endodontia todo mês. Está lá } \\
\text { no meu contrato, eu assinei e os prefeitos assinaram. Então, em relação a } \\
\text { isso, por ter esse documento, tudo é muito organizado. Eu tenho uma coisa } \\
\text { a seguir, um manual. Na prefeitura é mais solto, e o consórcio por ter esse } \\
\text { contrato é mais organizado." (D01). }\end{array}$ \\
\hline $\begin{array}{l}\text { Estratégias e mecanismos } \\
\text { de implementação do } \\
\text { CEO-R }\end{array}$ & $\begin{array}{l}\text { "Aí chegou o CEO regional, e já é difícil para ele (município) manter o } \\
\text { CEO Municipal. Entâo, o que ele faz? Fecha o CEO Municipal. [...] Porque } \\
\text { chegou uma unidade com porte maior e não teve necessidade dos municípios } \\
\text { menores manterem os seus." (D01). } \\
\text { "Todo mundo quer ser atendido no CEO e na Policlínica, [...] é onde eles } \\
\text { se sentem bem acolhidos pelo SUS. [...] Então assim, nenhum prefeito quer } \\
\text { blefar contra isso, porque automaticamente vai respingar nele politicamente. } \\
\text { Se um prefeito diz que quer sair do consórcio, ele tem todo direito. Agora, } \\
\text { a repercussão político social será negativa. Então todo mundo cuida do } \\
\text { consórcio [...]." (D07). }\end{array}$ \\
\hline
\end{tabular}

Fonte: elaboração própria, 2018. 


\section{Discussão}

O estudo de Lima et al. (2012) assume que a regionalização é influenciada pelas características específicas da sua implementação e, ainda, pela direção que se deseja imprimir ao processo, onde o contexto se destaca como uma das dimensóes que condicionam a regionalização nos estados. Por sua vez, o contexto políticoinstitucional expressa a trajetória das políticas de saúde, o modo de funcionamento das instituições e o papel desempenhado pelas organizaçóes envolvidas.

A partir da percepção de que as dificuldades inerentes ao contexto comprometem maiores avanços na política de regionalizaçáo, os achados deste estudo reforçam a importância do fortalecimento do enfoque territorial no planejamento governamental e de políticas no desenvolvimento regional. Carvalho et al. (2017) chamam atenção para as influências decorrentes do grau de articulação existente entre os atores sociais nos espaços de governança.

Neste cenário, identificou-se que o processo de regionalização no estado do Ceará não apenas foi influenciado por esta articulação, mas favoreceu a conformação de arenas e relaçóes sistêmicas e interativas, que potencializaram a dinâmica de atuação dos atores, reforçando ainda mais as articulaçôes e mobilizando consensos. Por sua vez, as escolhas políticas se desdobraram na criação de estruturas regionais e no envolvimento de atores sociais que favoreceram a continuidade e reduziram possibilidades de alteraçóes radicais no processo de implementação dos CEO no contexto da regionalização da saúde no estado do Ceará. Nesta perspectiva, a continuidade administrativa na gestão estadual também pode ser identificada como um fator favorável à manutenção da prioridade da regionalização na agenda política, garantindo sustentabilidade e continuidade das estratégias em curso.

A partir das concepçóes de Viana et al. (VIANA; LIMA, 2011), foi possível identificar um padrão de governança tipo coordenada-cooperativa estabelecida no âmbito estadual, caracterizada pelo estabelecimento de uma direção para o processo de regionalização com definição de metas e objetivos específicos e por uma relação de cooperação entre os atores, principalmente os governamentais. No Ceará, destaca-se que a notória e relevante trajetória dos atores, tanto na gestáo quanto na assistência do SUS, contribuiu de forma expressiva para a superação dos conflitos de interesses em prol do consenso em torno da regionalização. 
Considerando a implantação da rede regionalizada de atenção à saúde como condição para levar o cuidado à saúde bucal a um novo patamar de atenção e assistência no estado, no ciclo das políticas públicas o documento marco (CEARÁ, 1998) representou uma "janela de oportunidades" para a entrada da saúde bucal na agenda de prioridades da política de saúde estadual, impedindo a construção de um processo, por vezes, descolado da rede de atenção, conforme observado nos achados de Mello et al. (2014) na Região de Saúde da Grande Florianópolis, Santa Catarina.

Outro aspecto fundamental na experiência do Ceará foi caracterizado pela sequência temporal de eventos que demarcaram a política de saúde bucal no estado. A iniciativa dos Centros de Especialidades Odontológicas Regionais antecedeu o processo de implantação de serviços especializados sob gestão dos municípios. Isso permitiu estruturar a rede de atenção à saúde bucal no estado independentemente de iniciativas municipais isoladas, geralmente induzidas pela lógica de um modelo assistencial em odontologia baseada estritamente no consumo de tecnologias especializadas.

Segundo Chaves e Silva (2007), ainda que a descentralização da gestão dos serviços de saúde bucal pelas diretrizes nacionais tenha gerado aumento significativo de repasses de recursos financeiros para a área, os melhores resultados foram observados em cenários com projetos de reorganização das práticas de saúde, de capacidade técnica e governabilidade. Nesta perspectiva, o modelo dos Consórcios Públicos em Saúde destacou-se como um importante instrumento de gestáo intergovernamental, permitindo o apoio e o fortalecimento da gestão estadual, ainda que também suscetível interferências de ondem político-partidária. Considerando que maior ingerência local foi observada nos momentos de maior distanciamento da SESA-CE no acompanhamento dos municípios, os achados deste estudo corroboram o papel estratégico da esfera estadual na mediação de conflitos e ingerência da política local que poderiam minar a lógica cooperativa na construção de redes intermunicipais.

No âmbito das experiências de consorciamento para gestão de serviços de média complexidade no país, Figueiredo, Goes e Martelli (2016) identificam que esse arranjo institucional ainda é incipiente para os CEO, considerando que a maioria dos municípios não apresenta o movimento regular de associação com os demais da regiáo (SANTOS, 2017), de modo a organizar a oferta de atendimentos odontológicos especializados regionalmente. 
Apesar de os CPS não representarem uma novidade no país (AMARAL; BLATT, 2011), sua implementação no estado do Ceará caracterizou-se pela grande escala, pela gestão intergovernamental (municipal e estadual) e pelo esquema de retenção e repasse de recursos financeiros, sendo este um importante condicionante no processo de implementação dos CEO-R. Nessa lógica, os CPS fortaleceram a regionalização e apontaram caminhos alternativos à municipalização atomizada, sobretudo na prestação de atenção especializada (ALMEIDA et al., 2019), reforçando a importância de arranjos cooperativos e corresponsabilização pela condução da política.

Assim como identificado por Goya et al. (2016), os CPS estabeleceram instâncias próprias para a tomada de decisão e condução da gestão de equipamentos de saúde, de forma a constituir novas fronteiras de governança no sistema do estado, expostas às influências político-partidárias - na centralidade da figura do prefeito, e econômicas - no contexto de subfinanciamento do SUS. Neste cenário, Marchildon (2016) destaca a importância das instâncias/atores regionais, estruturas descentralizadas estaduais, como mediadores dos processos de organização e de acompanhamento do desempenho da rede de atenção à saúde. Reforça-se que o cuidado à saúde bucal deve ocupar espaço nas discussóes sobre a estruturação das redes regionalizadas, sendo fundamental a participação de seus profissionais e gestores nas instâncias que agregam poder decisório.

De maneira geral, ainda que os estudos de Albuquerque (2013) e Chaves (2016) destaquem as dificuldades impostas por um desenho descentralizador da política (LEVCOVITZ; MACHADO; LIMA, 2001) quanto à cooperação intergovernamental e aos avanços na consolidação da integração regional dos serviços do SUS, a implementação dos CEO-R consorciados na trajetória de regionalização da saúde no Ceará favoreceu o protagonismo do estado no planejamento e na coordenação das redes assistenciais regionalizadas.

\section{Considerações finais}

Os resultados deste estudo apontam que a inserção da saúde bucal no contexto da regionalização da saúde, liderada pela Secretaria da Saúde do Estado, configurouse como condicionante fundamental para o êxito da implementaçáo dos CEO regionais no Ceará. Ao mesmo tempo, a experiência dos CEO-R colaborou para fortalecer e aumentar o escopo da regionalização da saúde no estado, corroborando 
um conjunto de evidências produzidas por outras investigaçóes (ANDRADE, 2016; PINTO, 2014; VIEIRA, 2014; GOIA et al., 2016; ALMEIDA et al., 2019). A institucionalidade da atenção à saúde bucal no estado também esteve condicionada pelo aumento do acesso às açóes e serviços especializados em saúde bucal através dos Consórcios Públicos de Saúde.

Diante de ingerências político-partidárias e constrangimentos impostos pelo subfinanciamento do SUS, reforça-se a importância do papel da gestão estadual na condução das políticas de saúde e das redes regionalizadas.

Por fim, a experiência do estado do Ceará na implementação dos CEO aporta elementos importantes para a discussão, aperfeiçoamento e sustentabilidade da própria Política Nacional de Saúde Bucal, com vistas a fortalecer e consolidar o direito ao acesso integral à saúde no SUS pela via do fortalecimento dos arranjos regionais. ${ }^{1}$

\section{Agradecimentos}

Ao CNPq, pela bolsa do Programa de Pós-Doutorado Júnior (Processo 150036/2017-5), concedido à segunda autora, cujos recursos apoiaram a realização do estudo. L. D. Lima é bolsista de produtividade do CNPq.

\section{Referências}

ALBUQUERQUE, M. V. O enfoque regional na politica de saúde brasileira (2001-2011): diretrizes nacionais e o processo de regionalização nos estados brasileiros. Tese (Doutorado) Universidade de São Paulo. São Paulo, 2013. 289 p.

ALMEIDA, P. F. et al. Redes regionalizadas e garantia de atenção especializada em saúde: a experiência do Ceará, Brasil. Ciênc. saúde coletiva. Rio de Janeiro, v. 24, n. 12, p. 4527-4539, 2019. AMARAL, S. M. S.; BLATT, C. R. Consórcio intermunicipal para a aquisição de medicamentos: impacto no desabastecimento e no custo. Rev Saúde Pública. São Paulo, v. 45, n. 4, p. 799-801, 2011.

ANDRADE, E. M. Avaliação dos Centros de Especialidades Odontológicas Regionais gerenciados pelos Consórcios Públicos de Saúde do Ceará. Dissertação (Mestrado) - Universidade Federal de Pernambuco, Recife, 2016.

ANTUNES, J. L. F. et al. A saúde bucal na agenda de prioridades em saúde pública. Rev Saúde Pública. São Paulo, p. 50-57, 2016.

BARDIN, L. Análise de conteúdo. São Paulo: Ediçôes, 2011. 
BRASIL. Ministério da Saúde. Diretrizes da Política Nacional de Saúde Bucal. Brasília: Ministério da Saúde, 2004.

. Ministério da Saúde. Secretaria de Atenção à Saúde. Departamento de Atenção Básica. A saúde bucal no Sistema Único de Saúde. Brasília: Ministério da Saúde, 2018. 350p.

BRAVO, M. et al. The healthcare system and the provision of oral healthcare in European Union member states. Part 2: Spain. British Dental Journal. v. 219, n. 11, Dec. 2015.

CARVALHO, A. L.; JESUS, W. L. ; SENRA, I. M. Regionalização no SUS: processo de implementação, desafios e perspectivas na visão crítica de gestores do sistema. Ciênc. saúde coletiva. Rio de Janeiro, v. 22, n. 4, p. 1155-1164, 2017.

CEARÁ. Secretaria da Saúde do Estado do Ceará. Plano Estadual de Saúde 2016-2019. Fortaleza: SESA, 2016.

. Conselho Estadual de Saúde. Resolução no 05/1998, de 21 de dezembro de 1998. Aprova o documento "Diretrizes de Reorganização da Atenção e dos Serviços do Sistema Único de Saúde do Ceará”. Diário Oficial do Estado, Fortaleza, 1998a.

. Conselho Estadual de Saúde. Resolução no 06/1998, de 21 de dezembro de 1998. Inclui os anexos de no 07 e 08. Diário Oficial do Estado, Fortaleza, 1998b.

CHAVES, S. C. (Org.) Politica de saúde bucal no Brasil: teoria e prática. Salvador: EDUFBA, 2016. 377p.

CHAVES, S. C. et al. Avaliação da oferta e utilização de especialidades odontológicas em serviços públicos de atenção secundária na Bahia, Brasil. Cad. Saúde Pública. Rio de Janeiro, v. 27, n.1, p.143-154, 2011.

. Política Nacional de Saúde Bucal: fatores associados à integralidade do cuidado. Rev. Saúde Pública. Rio de Janeiro, v. 44, n. 6, p. 1005-1013, 2010.

CHAVES, S. C.; SILVA, L. M. Atenção à saúde bucal e a descentralização da saúde no Brasil: estudo de dois casos exemplares no Estado da Bahia. Cad. Saúde Pública. Rio de Janeiro, v. 23, n. 5, p. 1119-1131, 2007.

CONTARATO, P.C. Politica nacional e contexto local: uma análise da implementação da política de saúde bucal no Município de Vitória, do Espírito Santo. Dissertação (Mestrado) Escola Nacional de Saúde Pública Sergio Arouca, Fiocruz, Rio de Janeiro, 2011.

FIGUEIREDO, N.; GOES, P. S.; MARTELli, P. (Orgs.) Os Caminhos da Saúde Bucal no Brasil: um olhar quali e quanti sobre os Centros de Especialidades Odontológicas (CEO) no Brasil. Recife: Editora UFPE, 2016.

GOYA, N. et al. Regionalização da saúde: (in)visibilidade e (i)materialidade da universalidade e integralidade em saúde no trânsito de institucionalidades. Saúde Soc. São Paulo, v. 25, n. 4, p. 902-919, 2016. 
LAVERTY, L.; HARRIS, R. Can conditional health policies be justified? A policy analysis of the new NHS dental contract reforms. Social Science \& Medicine, n. 207, p. 46-54, 2018.

LEVCOVITZ, E.; MACHADO, C. V.; LIMA, L. D. Política de saúde nos anos 90: relações intergovernamentais e o papel das normas operacionais básicas. Ciênc. saúde coletiva. Rio de Janeiro, v. 6, n. 2, p. 269-291, 2001.

LIMA, L. D.; VIANA, A. L. D. Descentralização, regionalização e instâncias intergovernamentais no Sistema Único de Saúde. In: VIANA, A. L. D.; LIMA, L. D. (Orgs.). Regionalização e relaçôes federativas na politica de saúde do Brasil. Rio de Janeiro, 2011. p. 39-63.

LIMA, L. D. et al. Regionalização e acesso à saúde nos estados brasileiros: condicionantes históricos e político-institucionais. Ciênc. saúde coletiva. Rio de Janeiro, v. 17, n. 11, p. 2881-2892, 2012.

MAIN, P.; LEAKE, J.; BURMAN, D. Oral health care in Canada-a view from the trenches. $J$ Can Dent Assoc., v. 72, n. 4, p. 319, May 2006.

MARCHILDON, G. P. Regionalization: What Have We Learned? Health Papers, v. 16, n. 1, p. 8-15, 2016.

MELLO, A. L. et al. Saúde bucal na rede de atenção e processo de regionalização. Ciênc. saúde coletiva. Rio de Janeiro, v. 19, n. 1, p. 205-214, 2014.

NARVAl, P. C.; FRAZÃO, P. Saúde bucal no Brasil: muito além do céu da boca. Rio de Janeiro: Editora Fiocruz, 2008. 148p.

PEGON-MACHAT, E. et al. The healthcare system and the provision of oral healthcare in: UE Member States: France. British Dental Journal. v. 220, n. 4, Feb. 2016.

PINTO, V. P. Avaliaçáo da acessibilidade ao Centro de Especialidades Odontológicas de abrangência macrorregional de Sobral, Ceará, Brasil. Ciênc. saúde coletiva. Rio de Janeiro, v. 19, n. 7, p. 2235-2244, 2014.

SANTOS, L. Região de saúde e suas redes de atenção: modelo organizativo sistêmico do SUS. Ciênc. saúde coletiva. Rio de Janeiro, v. 22, n. 4, p. 1281-1289, 2017.

SILVA, C. R. et al. Dificuldade de acesso a serviços de média complexidade em municípios de pequeno porte: um estudo de caso. Ciênc. saúde coletiva. Rio de Janeiro, v. 22, n. 4, p. 1109$1120,2017$.

VIANA, A. L.; LIMA, L. D. (Orgs.). Regionalização e relações federativas na política de saúde do Brasil. Rio de Janeiro: Contra Capa, 2011.

VIEIRA, L. B. Política nacional e contexto local: a Atenção à Saúde Bucal em municípios do estado do Ceará. Tese (Doutorado) - Escola Nacional de Saúde Pública Sérgio Arouca, Fiocruz, Rio de Janeiro, 2014.

VIANA, A. L. et al. O processo de regionalização no Brasil: influência das dimensóes políticas, estrutura e organização. Rev. Bras. Saúde Matern. Infant. Recife, n. 17, supl. 1, p. 45-S61, out. 2017. 


\section{Nota}

${ }^{1}$ C. L. Silva Junior: concepção e delineamento do estudo, análise e interpretação dos dados, redação e revisão do artigo e aprovação da versão final. P. F. de Almeida: concepção e delineamento do estudo, análise e interpretação dos dados, revisão crítica do artigo e aprovação da versão final. M. T. Martins Filho: interpretação dos dados, revisão crítica do artigo e aprovação da versão final. L. D. de Lima: análise e interpretação dos dados, revisão crítica do artigo e aprovação da versão final. 


\section{Abstract}

\section{Implementation of Dental Specialty Centers in the context of health regionalization in the state of Ceara, Brazil}

The study aims to analyze the regional implementation of the Dental Specialty Centers (CEO) in the state of Ceara, Brazil. It is an evaluative research based on a case study with a qualitative approach. Semi-structured interviews were conducted with 13 key informants from management and academia, complemented by document analysis and non-participant observation, in 2018. The content analysis considered the regionalization process and the trajectory of oral health policy in the state. There was a strong induction and leadership of the State Department of Health, which allowed the insertion of oral health in the process of regionalization of health care. There was a large-scale implementation of Regional CEOs, as opposed to the municipalist guideline induced by federal policy. The following stand out as facilitating factors: the expertise of the state actors involved in conducting the policy, the anticipation of the state oral health policy to national guidelines and the implementation of the Public Health Consortia responsible for organizing and offering specialized services in health regions. The experience of Ceara suggests the importance of the regional approach for strengthening and consolidating full access to oral health within the scope of the Unified Health System.

> Keywords: oral health; health public policy; Regional Health Planning; Dental specialties; Brazil. 